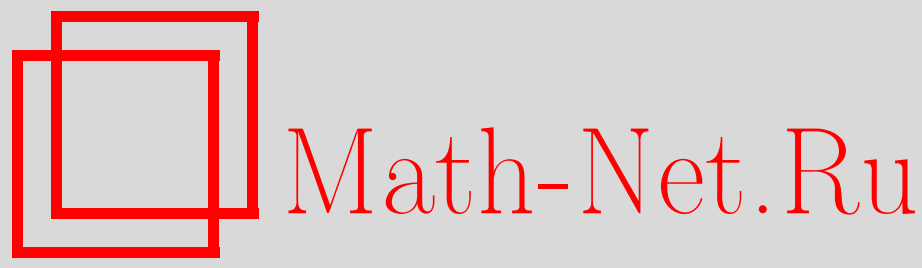

Ю. В. Павлов, О точных решениях и рождении частиц неконформных скалярных полей в однородных изотропных космологических моделях, ТМФ, 2013, том 174, номер 3, 504-512

DOI: https://doi.org/10.4213/tmf8393

Использование Общероссийского математического портала Math-Net.Ru подразумевает, что вы прочитали и согласны с пользовательским соглашением http: //www.mathnet.ru/rus/agreement

Параметры загрузки:

IP : 54.89 .56 .158

26 апреля 2023 г., 12:54:10

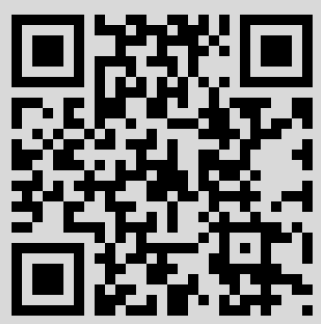




\title{
ФИЗИКА
}

Том 174, № 3

март, 2013

(C) 2013 г.

\author{
Ю. В. Павлов ${ }^{* \dagger}$
}

\section{О ТОЧНЫХ РЕШЕНИЯХ И РОЖДЕНИИ ЧАСТИЦ НЕКОНФОРМНЫХ СКАЛЯРНЫХ ПОЛЕЙ В ОДНОРОДНЫХ ИЗОТРОПНЫХ КОСМОЛОГИЧЕСКИХ МОДЕЛЯХ}

\begin{abstract}
Решена задача описания масштабных факторов однородного изотропного пространства-времени, при которых точные решения для скалярного поля с неконформной связью с кривизной могут быть получены из решений для поля с конформной связью переопределением массы и импульса. Представлены явные выражения в виде абелевых интегралов для зависимости времени от масштабного фактора в этих случаях. Получено точное решение для скалярного поля со связью с кривизной типа Гаусса-Бонне и показано, что соответствующие неконформные вклады могут быть доминирующими при рождении частиц гравитационным полем.
\end{abstract}

Ключевые слова: квантовая теория в искривленном пространстве-времени, рождение частиц гравитационным полем, скалярное поле, точные решения.

DOI: $10.4213 / \operatorname{tmf} 8393$

\section{1. ВВЕДЕНИЕ}

Квантовые эффекты в искривленном пространстве-времени, в частности рождение частиц гравитационным полем, могут иметь важные приложения в космологии и астрофизике [1], [2]. Исследования квантовой теории в гравитационном поле интенсивно проводятся с 70-х годов прошлого столетия. Особенно подробно изучен случай конформно-связанных полей в однородном изотропном пространстве-времени. Менее изучен случай неконформной связи скалярного поля с кривизной. Вместе с тем неконформные вклады могут быть доминирующими и в эффекте рождения частиц, и в величине вакуумных средних тензора энергии-импульса, определяющих обратное влияние квантованного поля на метрику пространства-времени (см., например, работы [3], [4]). До настоящего времени отсутствуют расчеты квантовых эффектов скалярного поля со связью с кривизной типа Гаусса-Бонне (ГБ).

* Институт проблем машиноведения РАН, Санкт-Петербург, Россия. E-mail: yuri.pavlov@mail.ru

†Лаборатория теоретической физики им. А. А. Фридмана, Санкт-Петербург, Россия 
В настоящей работе исследуется возможность получения точных решений для скалярных полей как с обычной неконформной связью с кривизной, так и со связью ГБ из решений для случая конформной связи в однородном изотропном пространстве-времени. Дается обзор масштабных факторов, допускающих получение точных решений для неконформных полей. Приведено точное решение для скалярного поля со связью с кривизной ГБ-типа и анализируется рождение частиц в такой модели. Расчеты рождения частиц проводятся в рамках квантовой теории поля в искривленном пространстве-времени, когда квантованные материальные поля рассматриваются во внешнем классическом гравитационном поле, описываемом метрикой пространства-времени. Возможность применения такого полуклассического подхода следует отнести к широкой области между планковскими и комптоновскими характерными значениями длины, кривизны и плотности [1], [2].

В работе используется система единиц, в которой $\hbar=c=1$.

\section{2. СКАЛЯРНОЕ ПОЛЕ В ОДНОРОДНОМ ИЗОТРОПНОМ ПРОСТРАНСТВЕ}

Рассмотрим комплексное скалярное поле $\varphi(x)$ массы $m$ с лагранжианом

$$
L(x)=\sqrt{|g|}\left[g^{i k} \partial_{i} \varphi^{*} \partial_{k} \varphi-\left(m^{2}+V_{g}\right) \varphi^{*} \varphi\right]
$$

и соответствующим уравнением движения

$$
\left(\nabla^{i} \nabla_{i}+V_{g}+m^{2}\right) \varphi(x)=0,
$$

где $\nabla_{i}$ - ковариантные производные в $N$-мерном пространстве-времени с метрикой $g_{i k}, g=\operatorname{det}\left(g_{i k}\right), V_{g}$ - функция инвариантных комбинаций метрического тензора $g_{i k}$ и его частных производных. $V_{g}=0$ соответствует минимальной связи скалярного поля с кривизной. Случай, когда $V_{g}=\xi_{\mathrm{c}} R$, где $R$ - скалярная кривизна и $\xi_{\mathrm{c}}=$ $(N-2) /[4(N-1)]$, называют конформной связью с кривизной $\left(\xi_{\text {c }}=1 / 6\right.$ при $\left.N=4\right)$. Уравнение (2) конформно-инвариантно, если $m=0$ и $V_{g}=\xi_{\mathrm{c}} R$.

Произвольная $V_{g}$ приводит к наличию в метрическом тензоре энергии-импульса скалярного поля, а следовательно, и в уравнениях Эйнштейна производных от метрики третьего и более высоких порядков. Появление в уравнениях дополнительных членов с производными высших порядков, даже если коэффициенты при них малы, приводит к радикальному изменению теории. Если потребовать, чтобы метрический тензор энергии-импульса не содержал производных от метрики выше второго порядка, то в качестве $V_{g}$ допустима функция

$$
V_{g}=\xi R+\zeta R_{\mathrm{GB}}^{2}
$$

где $R_{\mathrm{GB}}^{2} \stackrel{\text { def }}{=} R_{l m p q} R^{l m p q}-4 R_{l m} R^{l m}+R^{2}$. Величина $R_{\mathrm{GB}}^{2}$ в четырехмерном пространстве совпадает с плотностью эйлеровой характеристики, которая согласно теореме ГБ является топологическим инвариантом (в $N=2$ соответствующая плотность пропорциональна $R$ ). Поэтому связь с кривизной вида (3), введенную в работе [5], естественно называть связью ГБ-типа. В низших размерностях $N=2,3$ новых эффектов от $\zeta \neq 0$ не возникает, поскольку здесь $R_{\mathrm{GB}}^{2}=0$. В $N=4$ при постоянном $\varphi(x)$ вклад в метрический тензор энергии-импульса от члена с $R_{\mathrm{GB}}^{2}$ отсутствует, 
так как соответствующая вариационная производная обращается в нуль [6]. Но при переменном $\varphi(x)$ вклад от таких слагаемых необходимо учитывать, если константа $\zeta$ размерности (масса) ${ }^{-2}$ отлична от нуля.

Метрику $N$-мерного однородного изотропного пространства-времени запишем в виде

$$
d s^{2}=d t^{2}-a^{2}(t) d l^{2}=a^{2}(\eta)\left(d \eta^{2}-d l^{2}\right),
$$

где $d l^{2}=\gamma_{\alpha \beta} d x^{\alpha} d x^{\beta}$ - метрика $(N-1)$-мерного пространства постоянной кривизны $K=0, \pm 1$. Полная система решений уравнения (2) в метрике (4) может быть найдена в форме

$$
\varphi(x)=a^{-(N-2) / 2}(\eta) g_{\lambda}(\eta) \Phi_{J}(\mathbf{x})
$$

где

$$
\begin{gathered}
g_{\lambda}^{\prime \prime}(\eta)+\Omega^{2}(\eta) g_{\lambda}(\eta)=0 \\
\Omega^{2}(\eta)=m^{2} a^{2}+\lambda^{2}-\Delta \xi a^{2} R+\zeta a^{2} R_{\mathrm{GB}}^{2} \\
\Delta_{N-1} \Phi_{J}(\mathbf{x})=-\left(\lambda^{2}-\left(\frac{N-2}{2}\right)^{2} K\right) \Phi_{J}(\mathbf{x}),
\end{gathered}
$$

$\Delta \xi=\xi_{\text {c }}-\xi$, штрих обозначает производную по конформному времени $\eta, J$ - набор индексов (квантовых чисел), нумерующих собственные функции оператора Лапласа-Бельтрами $\Delta_{N-1}$ в $(N-1)$-мерном пространстве. В метрике (4) выражения для скалярной кривизны и инварианта ГБ имеют вид [5]

$$
\begin{gathered}
R=a^{-2}(N-1)\left[2 c^{\prime}+(N-2)\left(c^{2}+K\right)\right], \\
R_{\mathrm{GB}}^{2}=a^{-4}(N-1)(N-2)(N-3)\left(c^{2}+K\right)\left[4 c^{\prime}+(N-4)\left(c^{2}+K\right)\right],
\end{gathered}
$$

где $c=a^{\prime} / a=\dot{a}(t)$.

В соответствии с методом диагонализации гамильтониана [1] (случай произвольной $V_{g}$ см. в работе [7]) функции $g_{\lambda}(\eta)$ должны удовлетворять следующим начальным условиям:

$$
g_{\lambda}^{\prime}\left(\eta_{0}\right)=i \Omega\left(\eta_{0}\right) g_{\lambda}\left(\eta_{0}\right), \quad\left|g_{\lambda}\left(\eta_{0}\right)\right|=\Omega^{-1 / 2}\left(\eta_{0}\right)
$$

Если квантованное скалярное поле находится в вакуумном состоянии для момента времени $\eta_{0}$, то плотность числа пар частиц, рожденных к моменту времени $\eta$, может быть вычислена (для случая $K=0$ ) по формуле [1]

$$
n(\eta)=\frac{B_{N}}{2 a^{N-1}} \int_{0}^{\infty} S_{\lambda}(\eta) \lambda^{N-2} d \lambda,
$$

где $B_{N}=\left[2^{N-3} \pi^{(N-1) / 2} \Gamma((N-1) / 2)\right]^{-1}, \Gamma(z)$ - гамма-функция,

$$
S_{\lambda}(\eta)=\frac{\left|g_{\lambda}^{\prime}(\eta)-i \Omega g_{\lambda}(\eta)\right|^{2}}{4 \Omega}
$$

Как показано в работе [8], $S_{\lambda} \sim \lambda^{-6}$ и интеграл в (12) сходится при $N<7$. 


\section{3. О ТОЧНЫХ РЕШЕНИЯХ ПРИ РАЗНЫХ ТИПАХ СВЯЗИ С КРИВИЗНОЙ}

Уравнение (6) с помощью подстановки $g(\eta)=e^{z(\eta)}$ сводится к уравнению Риккати общего типа для $v(\eta) \equiv z^{\prime}(\eta)$ :

$$
v^{\prime}(\eta)+v^{2}(\eta)+\Omega^{2}(\eta)=0,
$$

поэтому число масштабных факторов $a(\eta)$, допускающих точные решения, относительно невелико. В случаях, когда точное решение все же может быть найдено, оно выражается, как правило, через специальные функции: гипергеометрические, функции Бесселя и т. д. Ряд точных решений приведен в книгах [1], [2]. Краткий обзор масштабных факторов, допускающих точные решения, дан в работах [9], [10].

В однородном изотропном пространстве-времени для поля с конформной связью $\Omega^{2}=m^{2} a^{2}+\lambda^{2}$, и уравнение (6) выглядит проще, чем при неконформной связи. Это не исключает существования точных аналитических решений более сложного уравнения для неконформного скалярного поля. Но большинство известных точных решений для неконформного скалярного поля получают из решений для конформного поля заменой параметров массы и импульса.

Рассмотрим следующий вопрос: при каких масштабных факторах точное решение для скалярного поля с неконформной связью с кривизной может быть получено из решения для поля с конформной связью переопределением значений массы поля $m$ и безразмерного импульса $\lambda$ ?

Для скалярного поля с неконформной связью вида $\xi R$ это будет иметь место, как следует из выражения (7), если найдутся константы $\beta_{1}, \beta_{2}$ такие, что

$$
\Delta \xi R a^{2}=\beta_{1}+\beta_{2} a^{2} .
$$

Данное условие с учетом выражения (9) представляет собой обыкновенное дифференциальное уравнение второго порядка относительно $a(\eta)$, сводящееся к линейному уравнению первого порядка для функции $f(a)=a^{\prime 2}(\eta)$. Его решение может быть представлено в виде

$$
\int \frac{d a}{\sqrt{C_{0} a^{4-N}+C_{1} a^{2}+C_{2} a^{4}}}= \pm\left(\eta-\eta_{0}\right),
$$

где $C_{0}, C_{1}, C_{2}, \eta_{0}$ - произвольные вещественные постоянные, при которых $C_{0} a^{4-N}+$ $C_{1} a^{2}+C_{2} a^{4}>0$.

В общем случае, при $N>4$, интеграл (16) гиперэллиптический, и вычисление $a(\eta)$ сводится к задаче обращения гиперэллиптического интеграла [11]. Для $N=4$ получение $a(\eta)$ из формулы (16) представляет собой задачу обращения эллиптического интеграла [12]. В частных случаях, когда интеграл (16) является псевдоэллиптическим или $N=4, C_{2}=0$, он будет выражаться в элементарных функциях. Приведем примеры таких масштабных факторов.

Первый пример масштабного фактора дается соотношениями

$$
C_{1}=C_{2}=0, \quad a=a_{1} \eta^{2 /(N-2)}=a_{0} t^{2 / N}, \quad a^{2} R=(N-1)(N-2) K,
$$

$a_{1}, a_{0}=$ const. В $N=4$ при $K=0$ такой масштабный фактор соответствует исключительно важному с точки зрения приложений случаю радиационно-доминирующей 
вселенной. Решения известны для $N=4$, где они могут быть выражены через гипергеометрическую функцию Куммера, и для $N=6$, когда они выражаются через функции Бесселя [9], [13].

Второй пример масштабного фактора:

$$
C_{0}=C_{2}=0, \quad a=a_{1} e^{\alpha \eta}=\alpha t, \quad a^{2} R=(N-1)(N-2)\left(\alpha^{2}+K\right),
$$

$\alpha=$ const. При $\alpha=1, K=-1$ это вселенная Милна [14] (т. е. часть пространства Минковского в соответствующих координатах). Решение уравнения (6) через функцию Бесселя представлено в работе [9].

Третий пример масштабного фактора:

$$
\begin{gathered}
C_{0}=C_{1}=0, \quad C_{2}=H^{2}, \quad a=-\frac{1}{H \eta}=\frac{e^{H t}}{H}, \\
a^{2} R=(N-1)\left[N H^{2} a^{2}+(N-2) K\right],
\end{gathered}
$$

$H=$ const. При $K=0$ соответствующие координаты описывают часть вселенной де Ситтера. Решение уравнения (6) выражается через функцию Ханкеля [1].

Для $C_{0}=0$ решениями уравнения (16) также являются

$$
\frac{a_{1}}{\operatorname{ch}(\gamma \eta)}=a_{1} \sin \frac{\gamma t}{a_{1}}, \quad \frac{-a_{1}}{\operatorname{sh}(\gamma \eta)}=a_{1} \operatorname{sh} \frac{\gamma t}{a_{1}}, \quad \frac{a_{1}}{\cos (\gamma \eta)}=a_{1} \operatorname{ch} \frac{\gamma t}{a_{1}}
$$

где $\gamma=$ const. В четырехмерном пространстве-времени решениями уравнения (16) для $C_{1}^{2}=4 C_{0} C_{2}$ будут

$$
\begin{gathered}
a_{1} \operatorname{tg}(\gamma \eta)=a_{1} \sqrt{e^{2 \gamma t / a_{1}}-1}, \quad a_{1} \operatorname{th}(\gamma \eta)=a_{1} \sqrt{1-e^{-2 \gamma t / a_{1}}} \\
-a_{1} \operatorname{cth}(\gamma \eta)=a_{1} \sqrt{e^{2 \gamma t / a_{1}}+1}
\end{gathered}
$$

а для $C_{2}=0-$

$$
a_{1} \sin (\gamma \eta)=\sqrt{a_{1}^{2}-\gamma^{2} t^{2}}, \quad a_{1} \operatorname{sh}(\gamma \eta)=\sqrt{\gamma^{2} t^{2}-a_{1}^{2}}, \quad a_{1} \operatorname{ch}(\gamma \eta)=\sqrt{\gamma^{2} t^{2}+a_{1}^{2}} .
$$

Решения уравнения (6) с масштабными факторами (20), (21) выражаются через гипергеометрические функции. Уравнение (6) с масштабными факторами (22) сводится к (модифицированному) уравнению Матье.

Заметим, что если найдено решение уравнения (6) с масштабным фактором $a(\eta)$, то из него переопределением $\lambda$ может быть получено решение для масштабного фактора $\tilde{a}=\sqrt{a^{2}(\eta)+b^{2}}$, где $b=$ const.

При использовании координатного времени $t$ решения уравнения (15) могут быть представлены в виде

$$
\int \frac{d\left(a^{N / 2}\right)}{\sqrt{C_{0}+C_{1} a^{N-2}+C_{2} a^{N}}}= \pm \frac{N}{2}\left(t-t_{0}\right),
$$

$t_{0}=$ const. Для $N=4$ интеграл (23) выражается в элементарных функциях. Таким образом, в четырехмерном пространстве-времени зависимость координатного времени $t$ от масштабных факторов, при которых решения для скалярного поля 
со связъю с кривизной $\xi R$ могут быть получены из решений для поля с конформной связъю переопределением значений массы и импульса, должна выражаться в конечном виде через элементарные функции.

Рассмотрим случай скалярного поля со связью с кривизной ГБ (3) и будем считать, что $N \geqslant 4, \zeta \neq 0$. Точное решение уравнения (6) может быть получено из решения для поля с конформной связью переопределением значений $m$ и $\lambda$, если найдутся константы $\tilde{\beta}_{1}, \tilde{\beta}_{2}$ такие, что

$$
-\Delta \xi R a^{2}+\zeta R_{\mathrm{GB}}^{2} a^{2}=\tilde{\beta}_{1}+\tilde{\beta}_{2} a^{2} .
$$

Данное условие с учетом формул (9), (10) сводится к линейному неоднородному дифференциальному уравнению первого порядка для функции

$$
F(a)=\left(\dot{a}^{2}(t)+K-\frac{\Delta \xi a^{2}}{2 \zeta(N-2)(N-3)}\right)^{2} .
$$

Решая дифференциальное уравнение (24) относительно функции $a(t)$, получим выражение для зависимости масштабного фактора от координатного времени:

$$
\int\left[-K+\frac{\Delta \xi a^{2}}{2 \zeta(N-2)(N-3)} \pm \sqrt{D_{0} a^{4-N}+D_{1} a^{2}+D_{2} a^{4}}\right]^{-1 / 2} d a= \pm\left(t-t_{0}\right)
$$

где $D_{0}, D_{1}, D_{2}$ - произвольные вещественные постоянные, при которых данный интеграл вещественный.

В общем случае нахождение явного вида $a(t)$ есть обращение абелева интеграла (26). Приведем примеры для частных случаев:

$$
K=\Delta \xi=D_{1}=D_{2}=0, \quad a=\gamma t^{4 / N}, \quad R_{\mathrm{GB}}^{2}=0,
$$

точные решения для конформной связи здесь известны при $N=4,8,12$ (см. формулы (17), (18));

$$
K=D_{0}=D_{1}=0, \quad a=a_{0} e^{H t}, \quad R_{\mathrm{GB}}^{2}=N(N-1)(N-2)(N-3) H^{4}
$$

(см. формулы (19)), для $N=4$ функции (20) будут решениями (26) при $D_{1}^{2}=4 D_{0} D_{2}$.

Далее мы рассмотрим точное решение и рождение частиц для первого из масштабных факторов (20).

\section{4. РОЖДЕНИЕ ЧАСТИЦ В МОДЕЛИ $a_{1} \sin \left(\gamma t / a_{1}\right)=a_{1} / \operatorname{ch}(\gamma \eta)$}

Пространство-время с таким масштабным фактором эволюционирует между двумя сингулярностями $t=0(\eta=-\infty)$ и $t=T \equiv \pi a_{1} / \gamma(\eta=+\infty)$, если $\gamma^{2}+K \neq 0$, $N \geqslant 3$. В случае $\gamma=1, K=-1$ соответствующие координаты покрывают часть пространства де Ситтера 2 рода [15] с постоянной отрицательной кривизной $R=$ $-(N-1) N / a_{1}^{2}$.

Рождение частиц в такой модели рассматривалось ранее для конформной связи с кривизной в книге [1], случай связи вида $\xi R$ исследовался в работе [3]. Рассмотрим здесь рождение скалярных частиц со связью с кривизной ГБ-типа (3) в четырехмерном пространстве-времени. Из формул (9), (10) в $N=4$ получим

$$
R a^{2}=6\left(\gamma^{2}+K\right)-\frac{12 \gamma^{2}}{a_{1}^{2}} a^{2}, \quad a^{2} R_{\mathrm{GB}}^{2}=-\frac{24 \gamma^{2}}{a_{1}^{2}}\left[K+\gamma^{2}\left(1-\frac{a^{2}}{a_{1}^{2}}\right)\right],
$$


поэтому уравнение (6) принимает вид

$$
g^{\prime \prime}(\eta)+\left[\left(m^{2} a_{1}^{2}+\Delta \xi 12 \gamma^{2}+\zeta \frac{24 \gamma^{4}}{a_{1}^{2}}\right) \frac{1}{\operatorname{ch}^{2}(\gamma \eta)}+\lambda^{2}-6\left(\gamma^{2}+K\right)\left(\Delta \xi+\zeta \frac{4 \gamma^{2}}{a_{1}^{2}}\right)\right] g(\eta)=0
$$

Решение уравнения (30) с начальными условиями (11) при $\eta \rightarrow-\infty$ может быть получено из точного решения для конформно-связанного случая [1] переопределением значений массы $(m \rightarrow M)$ и импульса $(\lambda \rightarrow \Lambda)$ :

$$
M^{2}=m^{2}+\frac{12 \gamma^{2}}{a_{1}^{2}}\left(\Delta \xi+\zeta \frac{2 \gamma^{2}}{a_{1}^{2}}\right), \quad \Lambda^{2}=\lambda^{2}-6\left(\gamma^{2}+K\right)\left(\Delta \xi+\zeta \frac{4 \gamma^{2}}{a_{1}^{2}}\right),
$$

и имеет вид

$$
g(\eta)=\frac{e^{i\left(\Lambda \eta+\alpha_{0}\right)}}{\sqrt{\Lambda}} F\left(A, B ; C ; \frac{1+\operatorname{th}(\gamma \eta)}{2}\right),
$$

где $F(A, B ; C ; z)$ - гипергеометрическая функция, $\alpha_{0}$ - произвольная вещественная постоянная,

$$
A=\frac{1}{2}+\sqrt{\frac{1}{4}+\frac{M^{2} a_{1}^{2}}{\gamma^{2}}}, \quad B=\frac{1}{2}-\sqrt{\frac{1}{4}+\frac{M^{2} a_{1}^{2}}{\gamma^{2}}}, \quad C=1+i \frac{\Lambda}{\gamma} .
$$

Условие $\Omega^{2} \geqslant 0$, необходимое в методе диагонализации гамильтониана, будет выполнено для уравнения (30) при любых $\lambda, \eta$, если

$$
\left(\gamma^{2}+K\right)\left(\Delta \xi+\zeta \frac{4 \gamma^{2}}{a_{1}^{2}}\right) \leqslant 0, \quad m^{2} a_{1}^{2}+6\left(\gamma^{2}-K\right) \Delta \xi-24 \zeta K \frac{\gamma^{2}}{a_{1}^{2}} \geqslant 0
$$

В частности, $\Omega^{2} \geqslant 0$ при $K=0$ и

$$
-\zeta \frac{4 \gamma^{2}}{a_{1}^{2}} \geqslant \Delta \xi \geqslant-\frac{m^{2} a_{1}^{2}}{6 \gamma^{2}}
$$

Из асимптотики выражения (13) для точного решения (32) аналогично конформному случаю [1] получаем предельный (при $\eta \rightarrow+\infty)$ спектр рожденных частиц:

$$
S_{\lambda}=\frac{\cos ^{2}(\pi / 2) \sqrt{1+4 M^{2} a_{1}^{2} / \gamma^{2}}}{\operatorname{sh}^{2}(\pi \Lambda / \gamma)}
$$

Число рожденных пар частиц в лагранжевом объеме $a^{3}(t)$ при $K=0$ за цикл эволюции $t \rightarrow T$ согласно формулам (12), (31), (36) будет равно

$$
N=\frac{\gamma^{3}}{2 \pi^{5}} \cos ^{2} \pi \sqrt{\frac{1}{4}+\frac{m^{2} a_{1}^{2}}{\gamma^{2}}+12 \Delta \xi+24 \zeta \frac{\gamma^{2}}{a_{1}^{2}}} \int_{x_{0}}^{\infty} \frac{x \sqrt{x^{2}-x_{0}^{2}}}{\operatorname{sh}^{2} x} d x
$$

где

$$
x_{0}=\pi \sqrt{-6\left(\Delta \xi+\zeta \frac{4 \gamma^{2}}{a_{1}^{2}}\right)} \text {. }
$$




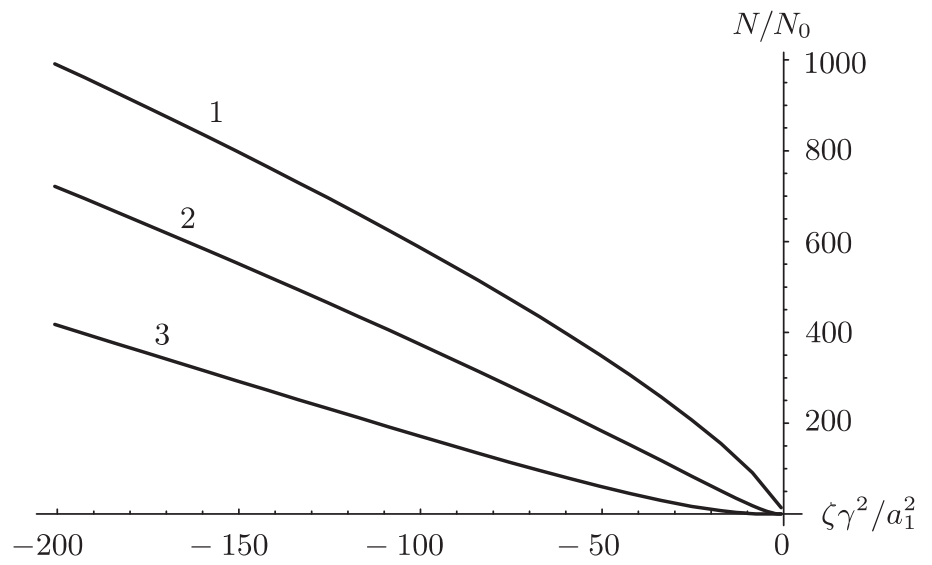

Рис. 1. Зависимость числа рожденных частиц от параметра $\zeta$ : кривая 1 $\Delta \xi=-1 / 6$, кривая $2-\Delta \xi=1$, кривая $3-\Delta \xi=3$.

Поскольку [16]

$$
\int_{0}^{\infty} \frac{x^{2}}{\operatorname{sh}^{2} x} d x=\frac{\pi^{2}}{6}
$$

с учетом формул (31), (35), (38)

$$
\Delta \xi=-\zeta \frac{4 \gamma^{2}}{a_{1}^{2}} \geqslant-\frac{m^{2} a_{1}^{2}}{6 \gamma^{2}} \Rightarrow N=\frac{\gamma^{3}}{12 \pi^{3}} \cos ^{2} \frac{\pi}{2} \sqrt{1+24 \Delta \xi+\frac{4 m^{2} a_{1}^{2}}{\gamma^{2}}} .
$$

При $\zeta=0$ формулы (36), (37) воспроизводят соответствующие результаты для случая скалярного поля со связью вида $\xi R[3]$, а при $\zeta=0=\Delta \xi-$ результаты для конформно-связанных частиц [1]. При $\Delta \xi=0$ формула (40) дает число пар конформно-связанных частиц, рожденных за цикл эволюции:

$$
N_{0}=\frac{\gamma^{3}}{12 \pi^{3}} \cos ^{2} \frac{\pi}{2} \sqrt{1+\frac{4 m^{2} a_{1}^{2}}{\gamma^{2}}}
$$

Если, при фиксированном $\Delta \xi$ параметр $\zeta \rightarrow-\infty$, то $M^{2} \rightarrow-\infty$, и число рожденных частиц неограниченно растет, как показано, например, для $m a_{1} / \gamma=1$ на Рис. 1.

Рождение частиц за цикл эволюции отсутствует, как следует из (36), если

$$
M^{2} a_{1}^{2}=n(n+1) \gamma^{2}, \quad n=0,1,2, \ldots .
$$

С учетом выражений (31) и неравенств (35) для $K=0$ рождения частиц за цикл эволюции нет, когда параметр $\zeta$ принимает значения

$$
\zeta_{n}=\frac{a_{1}^{2}}{24 \gamma^{2}}\left(n(n+1)-12 \Delta \xi-\frac{m^{2} a_{1}^{2}}{\gamma^{2}}\right), \quad n=0,1, \ldots \leqslant \sqrt{\frac{m^{2} a_{1}^{2}}{\gamma^{2}}+6 \Delta \xi+\frac{1}{4}}-\frac{1}{2}
$$

Таким образом, в зависимости от значения параметра $\zeta$ в рассмотренной модели рождение частиц может как неограниченно возрастать, так и отсутствовать 
вовсе. Следовательно, влияние параметра $\zeta$ связи скалярного поля с инвариантом ГБ может быть доминирующим, и его учет необходим при вычислении эффектов скалярных полей в искривленном пространстве-времени.

Благодарности. Автор благодарит проф. А. А. Гриба и участников семинара Лаборатории теоретической физики им. А. А. Фридмана за обсуждение работы. Исследование проведено в рамках сотрудничества с Copernicus Center for Interdisciplinary Studies, Краков, Польша, и поддержано грантом The John Templeton Foundation.

\section{Список литературы}

[1] А. А. Гриб, С. Г. Мамаев, В. М. Мостепаненко, Вакуумные квантовые эфбекты в сильных полях, Энергоатомиздат, М., 1988.

[2] Н. Биррелл, П. Девис, Квантованные поля в искривленном пространстве-времени, Мир, М., 1984.

[3] V.B. Bezerra, V. M. Mostepanenko, C. Romero, Modern Phys. Lett. A, 12:3 (1997), $145-154$.

[4] M. Bordag, J. Lindig, V. M. Mostepanenko, Yu. V. Pavlov, Internat. J. Modern Phys. D, 6:4 (1997), 449-463.

[5] Ю. В. Павлов, ТМФ, 140:2 (2004), 241-255.

[6] C. Lanczos, Ann. Math., 39:4 (1938), 842-850.

[7] Yu. V. Pavlov, Internat. J. Modern Phys., 17:6-7 (2002), 1041-1044, arXiv: gr-qc/0202041.

[8] Ю. В. Павлов, ТМФ, 126:1 (2001), 115-124.

[9] Ю.В. Павлов, "Рождение частиц в космологии. Точные решения", Квантовая теория и космология, Сборник статей, посвященный 70-летию профессора А. А. Гриба, ред. В. Ю. Дорофеев, Ю. В. Павлов, Изд-во Лаборатории им. А. А. Фридмана, СПб., 2009, 158-171.

[10] Ю.В. Павлов, Учен. зап. Казан. гос. ун-та. Сер. Физ.-матем. науки, 153:3 (2011), $65-71$.

[11] А.И. Маркушевич, Введение в классическую теорию абелевых функиий, Наука, М., 1979 .

[12] Г. Бейтмен, А. Эрдейи, Высшие трансцендентные функиии, т. 3: Эллиптические и автоморфные функиии, функиии Ламе и Матье, Наука, М., 1967.

[13] С. Г. Мамаев, В. М. Мостепаненко, А. А. Старобинский, ЖЭТФ, 70:5 (1976), 1577-1591.

[14] E. A. Milne, Nature, 130 (1932), 9-10.

[15] С. Хокинг, Дж. Эллис, Крупномасштабная структура пространства-времени, Мир, M., 1977.

[16] И.С. Градштейн, И.М. Рыжик, Таблицы интегралов, сумм, рядов и произведений, БХВ-Петербург, СПб., 2011. 